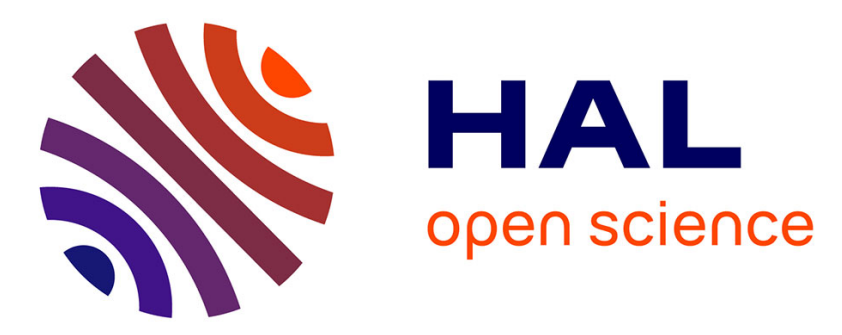

\title{
Enhancement of the Traffic Differentiation Architecture for WBAN based on IEEE 802.15.4
}

Sabri Khssibi, Adrien van den Bossche, Hanen Idoudi, Leila Saidane, Thierry Val

\section{> To cite this version:}

Sabri Khssibi, Adrien van den Bossche, Hanen Idoudi, Leila Saidane, Thierry Val. Enhancement of the Traffic Differentiation Architecture for WBAN based on IEEE 802.15.4. Wireless Personal Communications, 2018, 101 (3), pp.0. 10.1007/s11277-018-5775-5 . hal-02316840

\section{HAL Id: hal-02316840 \\ https://hal.science/hal-02316840}

Submitted on 15 Oct 2019

HAL is a multi-disciplinary open access archive for the deposit and dissemination of scientific research documents, whether they are published or not. The documents may come from teaching and research institutions in France or abroad, or from public or private research centers.
L'archive ouverte pluridisciplinaire HAL, est destinée au dépôt et à la diffusion de documents scientifiques de niveau recherche, publiés ou non, émanant des établissements d'enseignement et de recherche français ou étrangers, des laboratoires publics ou privés. 


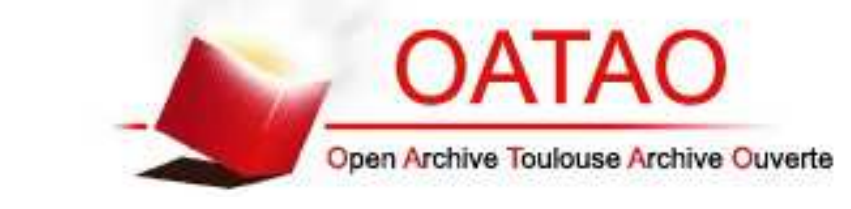

Open Archive Toulouse Archive Ouverte

OATAO is an open access repository that collects the work of Toulouse researchers and makes it freely available over the web where possible

This is an author's version published in:

http://oatao.univ-toulouse.fr/22415

\section{Official URL}

DOI : https://doi.org/10.1007/s11277-018-5775-5

To cite this version: Khssibi, Sabri and Van Den Bossche, Adrien and Idoudi, Hanen and Saidane, Leila and Val, Thierry Enhancement of the Traffic Differentiation Architecture for WBAN based on IEEE 802.15.4. (2018) Wireless Personal Communications, 101 (3). ISSN 0929-6212

Any correspondence concerning this service should be sent to the repository administrator: tech-oatao@listes-diff.inp-toulouse.fr 


\title{
Enhancement of the Traffic Differentiation Architecture for WBAN Based on IEEE 802.15.4
}

\author{
Sabri Khssibi ${ }^{1}$ (D) Adrien Van Den Bossche ${ }^{1} \cdot$ Hanen Idoudi $^{2} \cdot$ \\ Leila Azouz Saidane ${ }^{2} \cdot$ Thierry Val $^{1}$
}

\begin{abstract}
In the healthcare domain, Wireless Body Area Network has emerged as a vital technology that is capable of providing better methods to diagnose various hazardous diseases. The CANet projet is a project that proposes alternative monitoring solutions. This paper studies the possibility of transmitting different types of information through an IEEE 802.15.4 MAC layer that not supports the transmission of heterogeneous information. We have proposed an extension to the MAC layer which makes possible the transmission of various information types. This solution designed by "diffrentiation layer" uses a purge function to ensure the use of CAP and CFP by the same node in the same superframe and allows the differentiation between several information. Our results show that our solution is reliable under worst-case.
\end{abstract}

Keywords IEEE 802.15.4 - QoS · E-Health · WBAN · CANet project

Sabri Khssibi

khssibi@irit.fr

Adrien Van Den Bossche

bossche@irit.fr

Hanen Idoudi

hanen.idoudi@ensi.rnu.tn

Leila Azouz Saidane

leila.saidane@ensi.rnu.tn

Thierry Val

val@irit.fr

1 IRIT, UMR 5505 - CNRS, Universite de Toulouse, IUT Blagnac, 1 pl. Georges Brassens, BP60073, 31703 Blagnac Cedex, France

2 National School of Computer Science, University of Manouba, Campus of Manouba, 2010 Manouba, Tunisia 


\section{Introduction}

In recent years, the sensors networks applications field has been expanded and it has affected several areas, including (i) those in fire monitoring, toxic gas leaks...(ii) Identification and collection of environmental [1] information, various atmospheric indicators (iii) and military applications $[2,3]$.

The medical and assistance areas, also, took an interest in this emerging technology. The flexibility, low cost and rapid deployment characteristics of sensor networks allows its use discreetly in the patient environment or in any place if the patient requires continuous monitoring, especially for elderly $[4,5]$. Sensors network enables the access to any information types about the environment and health status.

Monitoring any suspicious change is a very advantageous point for this new technology. In economical level, monitoring an elderly person while keeping them at their usual living environment would reduce the charges of assistance. Also, the psychological and social side of the elderly will be ameliorated with the respect of his privacy and keeps its autonomy.

This new emerging technology is distinguished by ever-wider applications in the various areas of social life, accompanied by a great improvement in living conditions. These new applications allow scientific and industrial to define a new domain: eHealth field [6]. Several applications have been proposed, in this new field, like CANet project [7,8], intelligent racket $[9,10]$ and the Co-Robo Cane project [10]. These projects use sensors to collect information from the environment or the human body, then treat it locally or remote it to dedicated applications.

This paper is organized as follows. In Sect. 2, we present some related work in the QoS problem. In Sect. 3, we describe the main idea and the goals of CANet project. In addition, we present and analyze the main issue. Section 3.2 describe the IEEE 802.15.4 standard, particularly the PURGE and how to use it to resolve the detected problems. Section 4 presents the result of the performance analysis of our proposition. Finally, Sect. 5 presents our conclusions and future perspective.

\section{Related Work}

The emergence of WSNs as an important communication infrastructure regarding the realtime monitoring applications, such as health monitoring, increases the importance of QoS monitoring systems. QoS is keystones to achieve high levels of reliability and performance in WSNs [11-13]. Various QoS methods have been studied for WBAN: the works present in [14-18] and [19] adopt different scheduling, service differentiation, and novel hardware techniques to optimize the emergency transmission, packet latency, and power consumption. In [15], they propose an infrastructure for remote medical applications. In order to improve the delay and transmission time of critical vital signs, a differentiated service based on priority scheduling and data compression is presented. In this model, a patient server receives instructions from a remote hospital server and congures the patients WBAN accordingly. Each type of vital signal receives a priority level and it is transmitted according to its priority. In [16], they modify the framing structure of IEEE802.15.4 to remarkably reduce the packet delay of emergency alarms. There are also scheduling techniques utilizing prioritized retransmission to enhance WBAN QoS. In [17], presented a prioritization and congestion control services where they discriminate between different 
physiological signals and assign them to different priorities. In [18] have proposed a trafficadaptive MAC for handling emergency and on-demand traffic, in which a table is maintained to store the traffic patterns of the nodes. The authors modified the MAC superframe structure to include the configurable contention access period (CCAP); however, the rest of the superframe parts resemble the conventional IEEE 802.15.4 MAC. The traffic-adaptive MAC protocol described uses a traffic-based wakeup mechanism and a wakeup radio mechanism, to reliably accommodate various types of data. It utilizes the traffic information to enable low-power communication. In [19], a decentralized time synchronized channel swapping (DT-SCS) MAC has been proposed. The MAC protocol leverages pulsed-couple oscillators that simultaneously carry out the synchronization and desynchronization over multiple channels. The nodes randomly join a channel and automatically spread across the available channels. In [20], authors used the machine learning to improve the MAC performance. The proposed QL-based MAC protocol allows each node to determine better scheduling policy by learning the neighbor behavior and adapting the changes in the local traffic conditions.

There are a number of standards that could be used for building WBAN sytems like IEEE802.15.6 and IEEE.802.15.4. However, wireless UWB networks based on IEEE 802.15.4a offer the advantages of a large frequency range and low power spectral density, making it suitable for for healthcare and welfare related applications. The technology has matured sufficiently that it can be used to develop a real products. Similarly, a number of MAC protocols have been heavily researched and proposed based on IEEE 802.15.4. As WBAN technology has gained worldwide interest, considerable research efforts have been dedicated to proposing new MAC protocols based on 802.15.4 in order to satisfy the stringent requirements of WBANs.

To achieve the required QoS level many solution are proposed. In [21], these solutions are classified into 4 categories (Fig. 1).

This classification contains a different strategies used in IEEE $802.11 \mathrm{e}$ but can be used in WSN.

We had proposed a new classification, in [22], contains six sub-classes where every protocol can be classified under one of them. These sub-classes are under two main protocols classes (contention access period and contention free period).

To enhance the performances of IEEE 802.15.4, QoS algorithms (Fig. 2) were designed and can be divided into two sub-classes: algorithms intended to support QoS in CAP and others used to improve the CFP reservation technique [22].

The CAP class is composed of 3 types of algorithms [22]:

Fig. 1 Classification presented in [21]

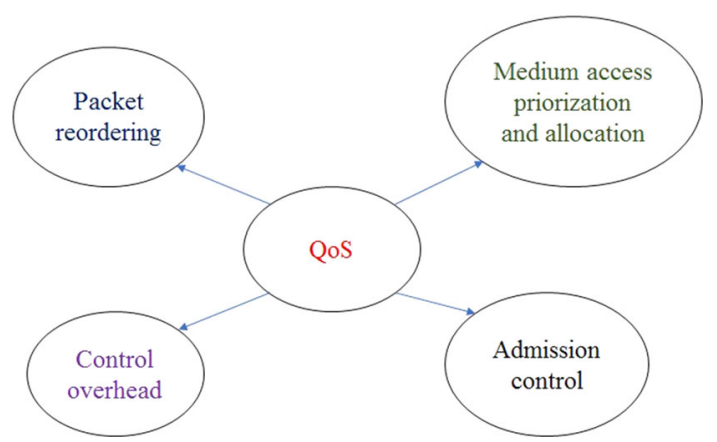




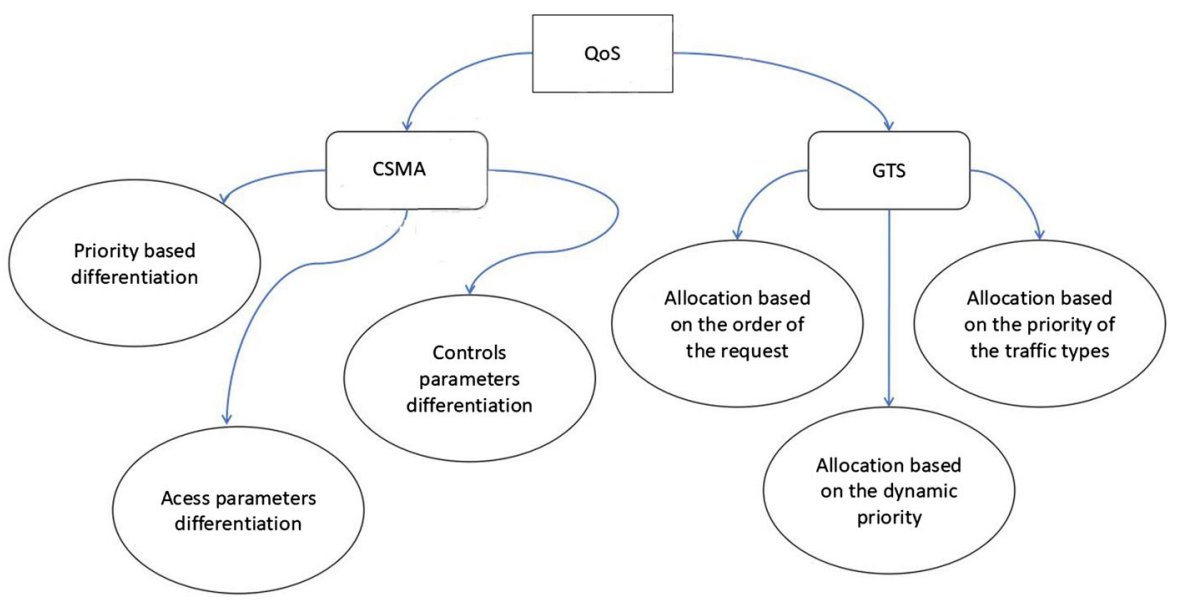

Fig. 2 Used protocols for traffics differenciation [22]

- Priority based differentiation: priorities may be assigned to nodes according to the importance of the information they handle or to classify the generated traffic [21, 23-25].

- Access parameters differentiation: different MAC layer parameters like CW, BackOffExponet and SIFS are tuned for different levels of priority. The aim is to expedite the transmission of packets that have a high priority [23, 26-30].

- Controls parameters differentiation: This class contains the QoS mechanisms that use parameters such as hop count, life-time of a packet and power control to manage the transfer of packets in the network. In this proposal, these parameters are used to differentiate between the existing traffics and to assign priorities [31-33].

CFP class includes algorithms which are proposed to resolve the problem of GTSs reservation in IEEE 802.15.4. It is divided into 3 parts:

- Allocation based on requests order: The coordinator reserves slots times to the nodes according to the order of their requests reception. If a node wants to reserve GTSs, it has to send a request to the coordinator, which contains the number of desired slots and the direction of the exchange (reception or transmission). Upon a request reception, the coordinator compares the number of requested GTSs with the number of available slots in the CFP period. If the number of requested slots is lower or equal to the number of available slots, the coordinator allocates the necessary slots to this node. In the other case, the coordinator rejects the request.

- Allocation based on the dynamic priority of nodes: GTS reservation depends on the priority assigned to each node. In [34], this priority changes dynamically according to the use of last reserved GTS. The priority change is followed by the reallocation of GTS in the network. The algorithm in [34] does not support any traffic differentiation. To meet this need, other protocols such as D-SeDGAM [35] allow the differentiation between services. This class suffers from several limits. For instance, if many nodes have a strong priority, they will monopolize the bandwidth. Also, this class does not take into account realtime traffics.

- Allocation based on traffic priority: In this third class, GTS allocation is adapted to real-time traffics. In the initial structure of IEEE 802.15.4 superframe, when a node 
wants to transmit data during CFP, it must send a GTS request and wait for the beacon. If the request was accepted, the node will transmit the packets only in the allocated period, which makes the current structure of the superframe inadequate to support the transmission of real-time data. In [27], the authors propose a new reservation algorithm based on an extension of the IEEE 802.15.4 GTS concept. They suggest to eliminate the inactive period, then SD becomes equal to BI. Other modifications are proposed in the superframe structure. Therefore, it supports the concept of multi-user at the time of communication.

This class does not take into account the energy consumption and allows the treatment of only one type of traffic.

\section{Differentiation Layer}

Many solutions are proposed to differentiation WBAN traffics (see related works section), but these solution are a many problems and request a major modification in the standard. We chose to use the IEEE802.15.4 instead of IEEE802.15.6 to have more bandwidth flexibility. In fact, IEEE802.15.6 is works in Narrow and UWB band contrary to IEEE802.15.4 that works in ISM, Narrow and ISM bandwidth. Althought th IEEE802.15.6 has a many drawbacks when it comes to using UWB. It ignores several limitations in the implementation of the tranceiver [36]. its assumes the use of UWB trancerver at the end node. The implementation of the UWB receiver of the IEEE802.15.6 involves a complex hardware designing and it ignores the optimization of the UWB transmit power control [36]. On other hand IEEE802.15.4, specially IEEE802.15.4a, is the most discussed and adopted standard for UWB. It have been the base of many MAC-UWB implementation. The performance of IEEE802.15.4a have been studied in many research paper. And they are shows that the MAC protocol of IEEE802.15.4a performs better in terms of throughtput and energy consuption for WBANs that contain a large number of sensors nodes [36].

Our work is particularly oriented to the CANet project case where the main aim is to offer oversight and monitoring without being intrusive. In order to be able to meet this exigence optimally, we were looking for an alternative for the conventional methods. The solution that we found is to integrate the different biometric sensors into a familiar equipment to the elderly. After a detailed investigation, the only equipment that can be matched with our criteria is the cane; where we can put all needed biometric sensors to collect information on the environment and health status of the elderly. For example, we can use a hand and outdoor temperature, a humidity, a heart rate, a pressure sensors and a wireless communication system...

The cane also has loudspeaker and microphone which give to the elderly the opportunity to live life to its fullest and to reach some family member or monitoring centers in case of an emergency, illness or loss. In Fig. 3, we suggest a set of biometric sensors that can be used in the CANet project. Sensors number and types used depends on the information types needed by medical staff.

Our cane must be connected to CMAP (Coordinator Mesh Access Point), in order to be able to transmit received information to the monitoring center. The most suitable topology of our network is the star topology (Fig. 4), where the transmission of information pass by the access point.

Many communication technologies can be used to establish a connection between the cane and the CMAP. A comparison was made in [37] between BLE, IEEE 802.15.4 and 


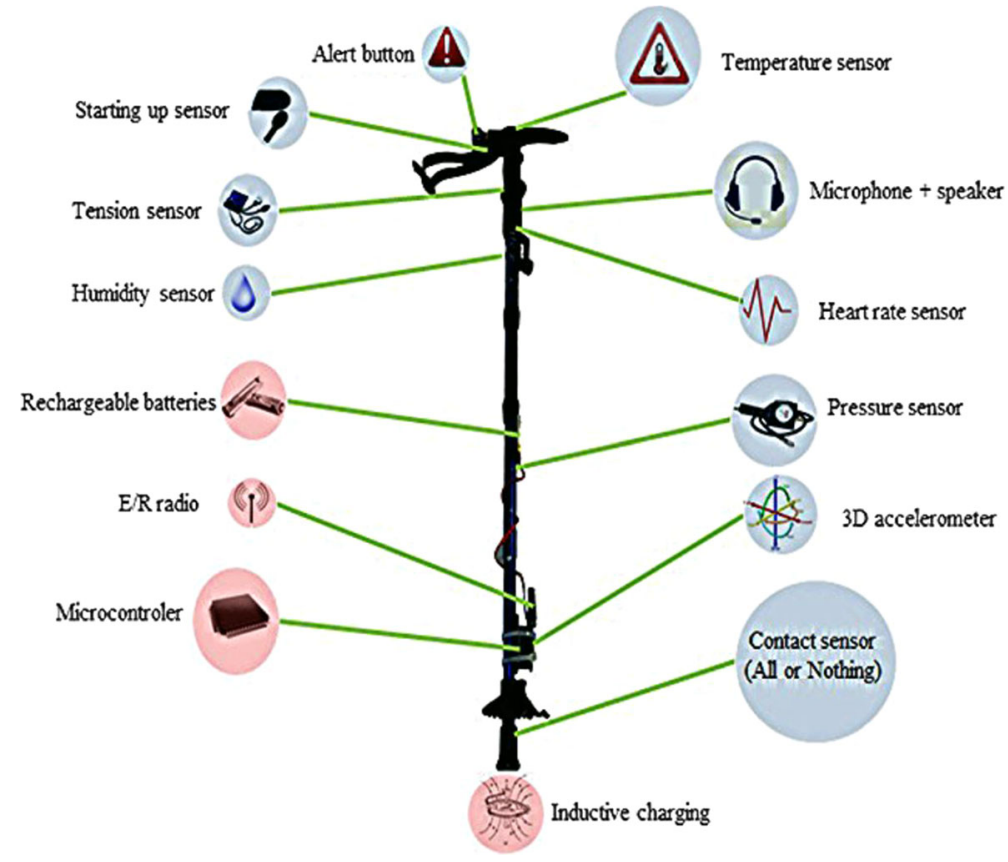

Fig. 3 Different biometric sensor types that can be used in CANet

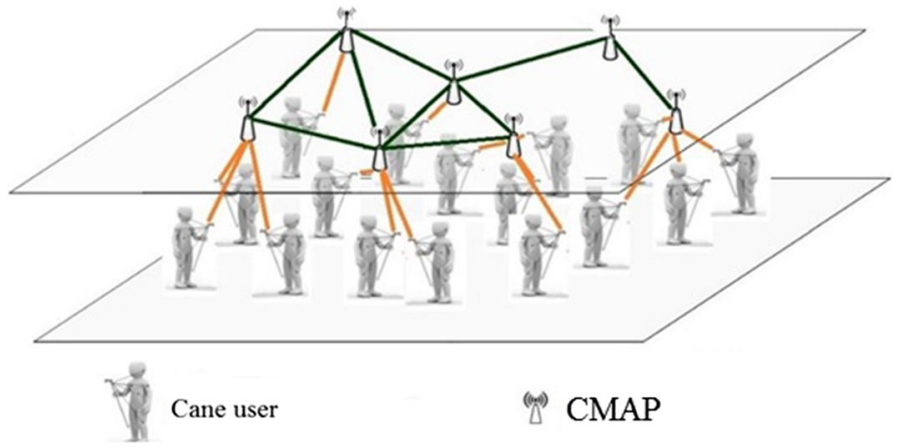

Fig. 4 Network architecture

IEEE 802.15.6. The authors recommend the use of IEEE 802.15.4 MAC protocol to communicate with the coordinator of the network.

This protocol represents a good choice in term of energy consumption and the QoS provided by the presence of two communication algorithms (CSMA and TDMA). But, it is not adapted to the network specific requirements in the context of CANet project. Indeed, IEEE 802.15.4 is made for the transmission of homogenous information. However, the generated traffics, in CANet project, are heterogeneous.

According to the study done in [37], the most relevant criteria common to all the CANet sensors are: periodicity, data rate, priority, and the real-time aspect: 
- Periodicity: Represents the duration of transmission cycle for each sensor. This duration varies according to the information type and the useful period which allows the detection of significant information.

- Pseudo-periodicity: It comes to traffics not always present and therefore unpredictable.

- Low periodicity: This category is intended for sensors generating data with high frequency (very short duration between two successive transmissions).

- Average periodicity: The period between two successive transmissions is of few minutes;

- Long periodicity: The duration between two successive transmissions exceeds that of the family of average periodicity.

- Rate:

- Low data rate: For data rates of few bytes per second at the most;

- Average data rate: This category is generally related to information for 2D or 3D representation such as GPS location data ;

- High data rate: Usually this kind of traffic contains data of a voice message and requires a large bandwidth.

- Priority:

- Low priority: For the data without particular requirements of transmission/ reception time or of QoS;

- High priority : includes the real-time data;

- Very high priority: This category is intended for alert data.

So IEEE 802.15.4 cannot satisfy all the applications requirements. In order to resolve this problem, we propose to add a DIFFERENTIATION service layer between the MAC and the application layers (Fig. 5).

Generated traffic are classified into three queues according to the rules pre-defined in the dispatcher sub-layer.

The Management sub-layer is responsible of the synchronization between the type of the queue where it takes the frame and the adequate period of transmission. This layer contains three queues:

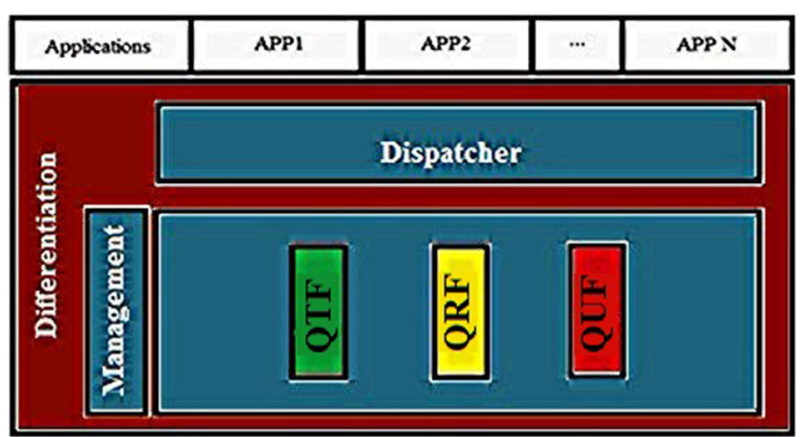

Fig. 5 Differentiation layer architecture 
- QTF (Queue of Tolerant Frame): Contains all frames that do not have a temporal requirement or sensitive to the lost frames.

- QRF (Queue of Real Frame): Contains all frames that have a temporal requirement.

- QUF (Queue of Urgent Frame): the frames of this queue will be transmitted in CFP and CAP periods.

This process makes the cane adapted to transmit different traffic in different periods in the same SD, but it is not not the most performant solution. In fact, IEEE 802.15.4 does not propose a mechanism to make the simultaneous transmissions in CAP and CFP periods possible.

The Fig. 6 shows an example that illustrates the transmission problem where we use two different medium access techniques by the same node. The MAC state can be:

- Busy by a frame has gotten from QTF: this state is represented by green color

- Busy by a frame has gotten from QRF: this state is represented by yellow color

- Free: this state is represented by white color; only in this state, the differentiation layer could transmit a frame from one of their queues to the MAC sublayer.

If the situation in the network is perfect, the nodes do not have problems to transmit their information. The MAC sublayer received frames from QTF in the CAP (represented by green color in the first and last superframe-see Fig. 6) and the frames received from QMF in the CFP (represented by yellow color in the first and the last superframe-see Fig. 6).

If the MAC sublayer couldn't transmit the frame, it keeps the frame until a successful access to the medium or the frame rejected due to failing to get access after three tries. This case is represented in the superframe number 3 and 4 in Fig. 6. The MAC sublayer keeps the frame and does not solicit other frames from differentiation layer. At this moment, the number of frames waiting in the QRF increases and probably the QRF reaches the maximum frame number supported rapidly, and then some frame going to be dropped.

If this deadlock situation repeats frequently, the QRF queue would overflow. Then, the cane is in situation where it loses frames of no tolerant application. This case is not appreciated because it has a big influence in the monitoring process.

Before we present our proposition we have to announce one rule that will influence our choice in the rest of the paper: The proposition must not require a significant modification in the IEEE 802.15.4 and will be like an extension to it. This rule can be crucial to the use of the proposition in the industrial solution in the future.

To achieve our goal we will study the solution provided by IEEE 802.15.4. After that, we will search how to use it to resolve our network problem.

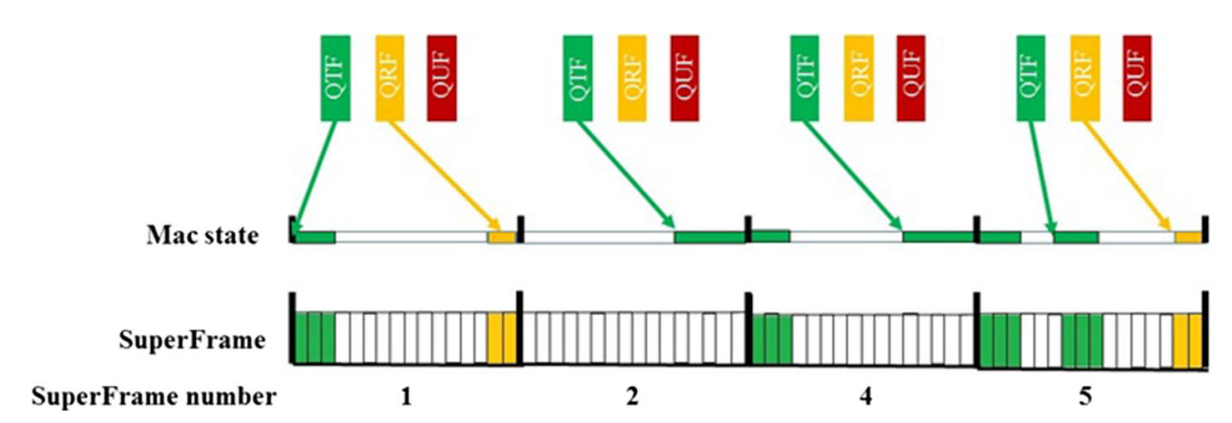

Fig. 6 Example of frame transmission 
In the next part of this paper, we will present the IEEE 802.15.4 standard particularly the PURGE function and our solution in order to ameliorate the differentiation layer performances.

\subsection{Differentiation Layer Enhancement}

\subsection{Purge Function}

The standard IEEE 802.15.4 defines the specifications of the MAC sub-layer and the PHY layer for Low-Rate Wireless Personal Area Networks (LR-WPAN). In addition, it defines two types of nodes:

- Reduce Function Device (RFD): does not implement the entire stack of IEEE 802.15.4, it has limited functionality

- Full Function Device (FFD): implements all the IEEE 802.15.4 specification and they can be coordinators or routers, etc.

IEEE 802.15.4 defined two communication modes:

- Non-beacon-enabled: In this mode, all nodes in the network use an un-slotted CSMA/ CA access mechanism

- Beacon-enabled: In this mode, all nodes are connected to one coordinator, which is responsible for the synchronization, guaranteed time slot (GTS) reservation and the beacon transmission.

In the rest of the paper we will focus only to the enabled-beacon mode.

Beacon frames are transmitted periodically by the coordinator. Beacon Interval (BI) is the time interval between beacon transmissions. The BI is divided into two periods: Superframe Duration (SD) and inactive period.

The SD is composed into two parts (Fig. 7):

- Contention Access Periods (CAP): The nodes use CSMA/CA access mechanism.

- Contention Free Periods (CFP): If a node needs to reserve GTSs in the CFP part, it will send a request command to the coordinator in the CAP. If the coordinator has free slots in the CFP, It would satisfy the requesting node. Therefore, the node transmits its information in the reserved GTS by using TDMA.

The standard defines some precious functions used by upper layers to manage the transmission in MAC layer.

Fig. 7 Trame composition

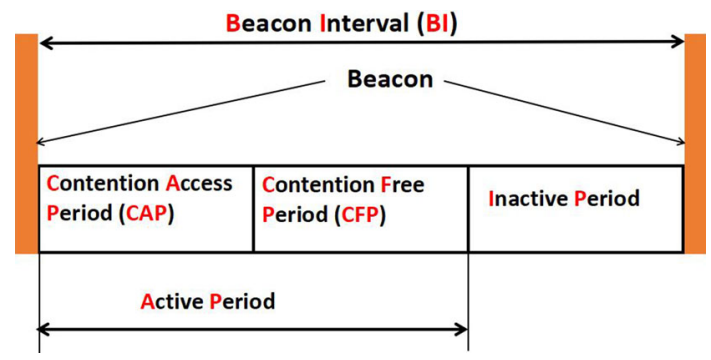


One of the most important functions is the PURGE. This function allows the next higher layer to purge a frame from the transaction queue of the MAC layer. Two primitives are defined:

- MCPS-PURGE.request: request to remove an MSDU from the transaction queue.

- MCPS-PURGE.confirm: allows the MAC sublayer to notify the next higher layer of the success to purge an MSDU from the transaction queue.

These two primitives make the next higher layer able to interrupt the transmission and delete the frame from the transaction queue.

\subsection{Proposition of Our Solutions}

The differentiation layer is unable to use two different access methods by the same MAC layer.

If the cane use the defined purge in the standard, the frame is going to be permanently deleted from the transmission queue and, in our case, the frame can be deleted only if we cannot transmit it with ordinary process defined by IEEE 802.15.4.

The Fig. 8 presents the amelioration that we propose. We had added three buffers that will contain a copy of transmitted frame to MAC sublayer. This copy is used, when the management sublayer request from the MAC layer to remove the frame from their transmission queue; then, the purge function is used to liberate the MAC sublayer. If the management sub-layer receives information from MAC layer indicates the success or the failure of the transmission, the frame in the buffer will be deleted.

In this part, we have introduced the project CANet and the main goals. We also have presented our solution to resolve the problem by the use of the purge function (provided by the standard) and how adapt the differentiation layer to this new feature.

In the next section, we will present our simulation and analysis of our proposition.

\section{Simulation, Results and Analysis}

In this part we are going to present our results and analysis. We have used the IEEE 802.15.4 model available in OMNet ++ simulator. First of all, we have verified if the implemented model in OMNet ++ follows the communications procedures described in the IEEE 802.15.4, we have compared their simulations result to other work made by

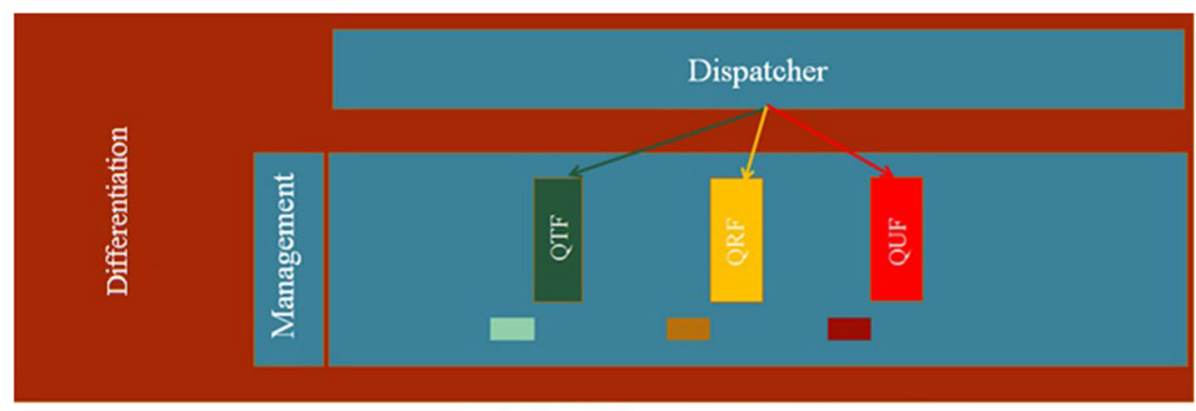

Defined buffer to different queue

Fig. 8 Modification in differentiation layer 
Table 1 Simulation parameters

\begin{tabular}{ll}
\hline Parameters & Values \\
\hline Topology & Star \\
Node numbers & $1,2,5,7$ \\
Traffic types & Heart rate sensor \\
& Accelerometer sensor \\
Queue length in differentiation layer & 20 frames \\
ACK management & \\
\multicolumn{1}{c}{ CAP } & With ACK \\
$\quad$ CFP & With ACK \\
& Without ACK \\
\hline
\end{tabular}

simulation and testbed. After that, we added some new functionality, defined in the standard, to the model. We also added our solution and re-checking the final model.

The Table 1 presents used parameters for our model. We have used star topology with one coordinator and $\mathrm{N}$ nodes, the node numbers are in the interval [ 1 ...7]. We consider only one collision area. The transmitted frame in CFP can be acknowledged or not acknowledged. In fact, the standard gives us the liberty to configure the ACK in the CFP.

In the network, each node can generate two different type of traffics that will emulate the heart rate (APP1) and accelerometer (APP2) sensors.

The APP1 tolerate latency and packet loss. However, the APP2 do not tolerate latency and even less packet loss. Then, the traffic generated by APP1 will be transmitted in CAP period and the other traffic (generated by APP2) will be transmitted in CFP period (cf. Table 2). The presented configuration is taked from real documentation to ensure that our approach is coherent with reality.

The use of ACK is importante to get a good performance. The standard gives us the possibility to use ACK in CFP period. For this reason, we present some simulation with and without ACK in CFP.

\subsection{Simulation of Transmissions Without Error}

In this scenario we have configured our simulator to have an ideal study environment. We dont have any transmission errors.

The Fig. 9 shows the difference between the percentages of received messages in three different configurations:

- with differentiation without purge function,

- without differentiation,

- and with differentiation with purge function.

Table 2 Application parameters

\begin{tabular}{lllll}
\hline Application & Sensor & Inter-messages & Message size & Transmission period \\
\hline APP1 & Heart rate & $20 \mathrm{~ms}$ & 3 Octets & CAP \\
APP1 & Accelerometer & $100 \mathrm{~ms}$ & 5 Octets & CFP \\
\hline
\end{tabular}




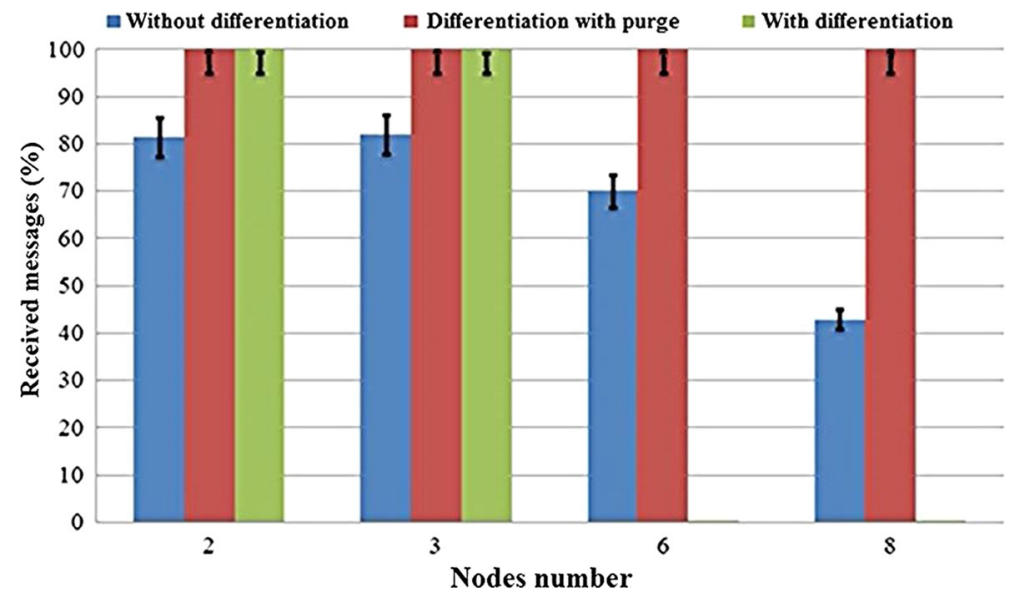

Fig. 9 The difference between the use of the purge and the other case in term of received messages in CFP

For 2 and 3 nodes in the network, the network is more preferment with differentiation layer than the other case. For 6 and 8 nodes, only the differentiation layer with the purge function kept the network performance in the same level (100\%). The simulations carried out without differentiation layer become better than the result founded when we use the differentiation layer without the purge function. Then we have a good results with our differentiation layer in term of performance and rate in the network.

The Fig. 10 shows the percentage of successful received messages when nodes used CAP in different configurations. We notice the existence of a slight improvement in the differentiation layer with purge use case.

After the simulation of our contribution in ideal situation, we include the possibility to have some transmission error, to test the robustness of our work in non-perfect environment where the transmission error occurs always in the layer 1 . In the following, we present some result of our simulation in the case of BER $=10^{-7}, 10^{-5}$ and $10^{-4}$.

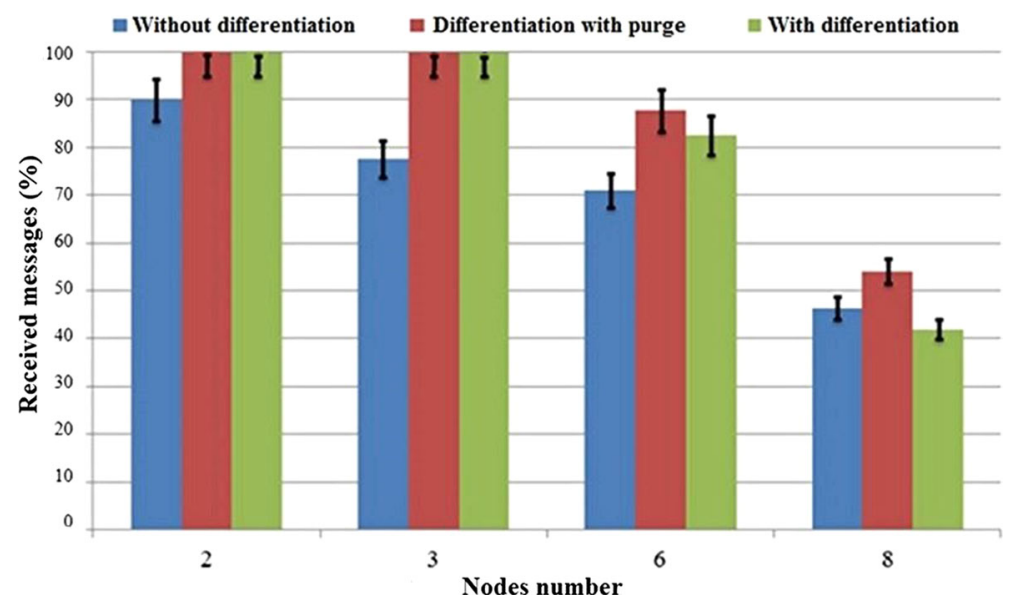

Fig. 10 Network performance in term of received messages in CAP 


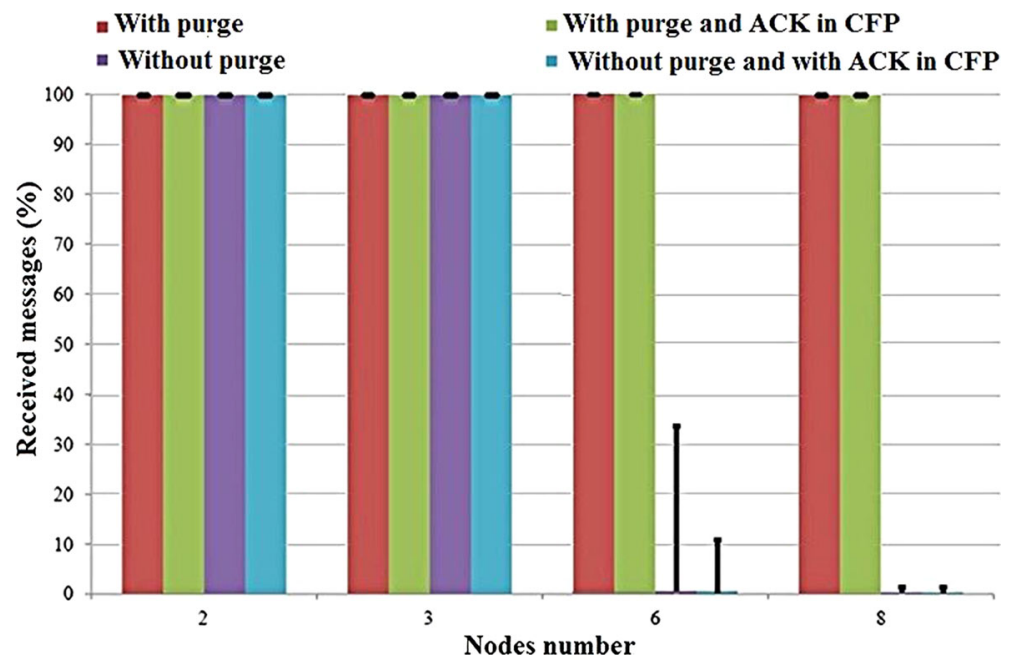

Fig. 11 Result of different simulations case

The obtained results show the benefit of the purge function use. With purge function the received messages reach $100 \%$ for the different network size contrary to other configuration (Fig. 11).

Purge function dramatically improved network performance. Failed frames decrease from a value greater than $1 \%$ to a value around of $0.8 \%$. This is due to the transmission parameters reset $(\mathrm{NB}, \mathrm{CW}$ and $\mathrm{BE}$ ) and a good transmission periods management (see Fig. 12).

The number of messages that do not fall within the queue (see Fig. 13 for FMT queue) increases slightly. This problem is related to the size of the queue and the configuration of the generation messages frequency in the application. It does not influence at all our solution performance but it reduces the number of received messages.

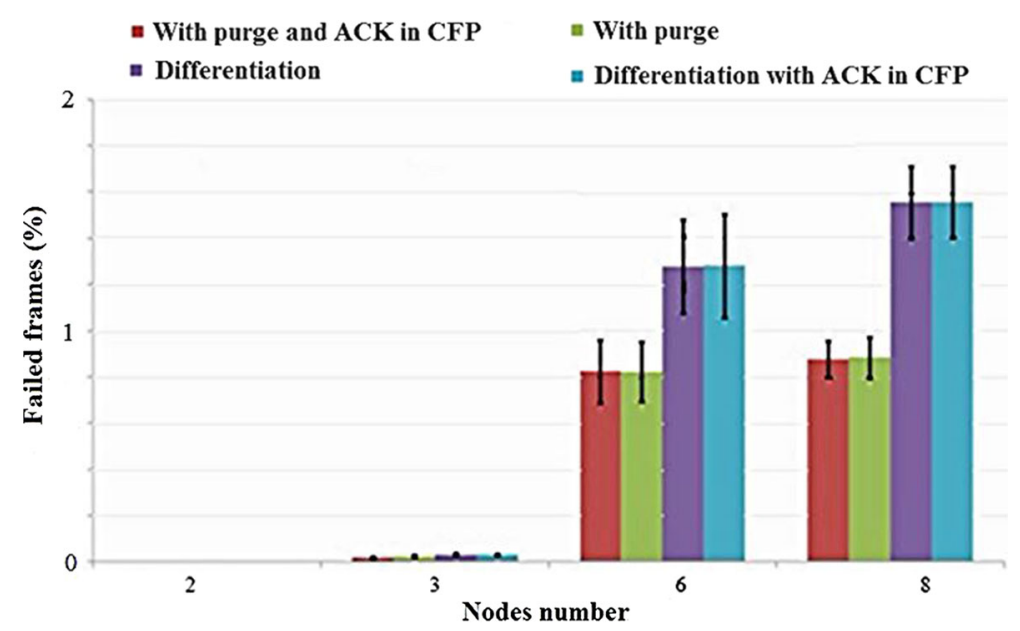

Fig. 12 Percentage of failed frames in different simulations case 


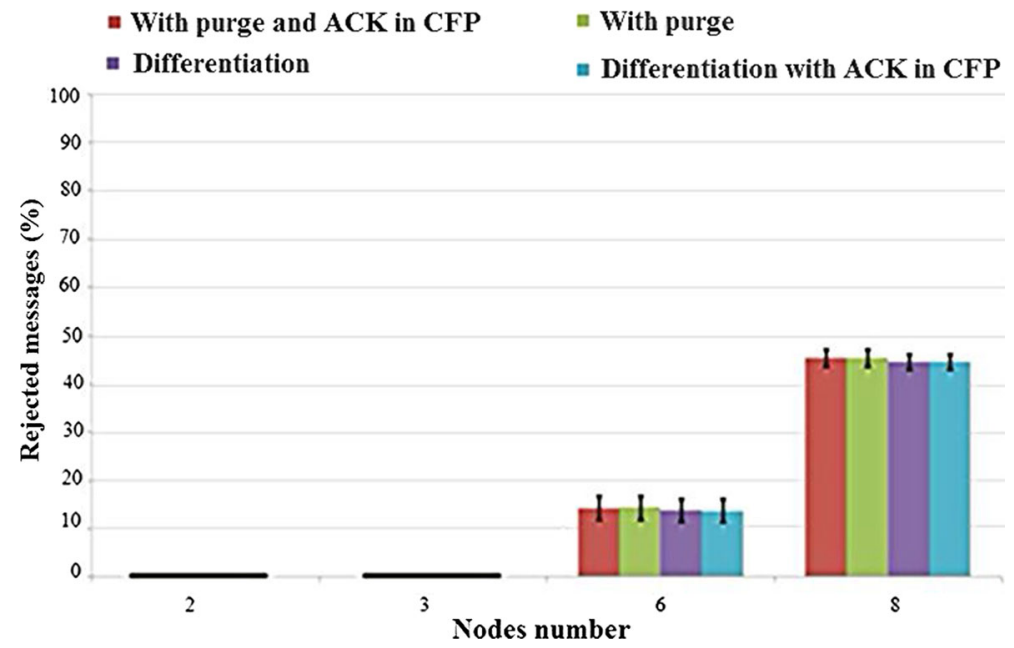

Fig. 13 Rejected messages percentage in different simulation cases

Finally, we noticed first of all that the use of the purge is necessary to keep the network performance in the case of large network. Secondly, there is a difference between the number of generated messages and received messages because of the technical issues related to the queue size and it is not related to our proposed solution.

The use of a perfect environment (BER equal to zero) does not reflect the real behavior of our solution. For this reason, we change the simulator configuration to add a more realistic BER (where BER can be: $10^{-7}, 10^{-5}$ and $10^{-3}$ ). The following simulations are performed only with the differentiation layer since purge provides better results than the other proposals. We use the same network configuration. Our objective is to test robustness of our solution in real environment.

The Fig. 14 shows that the differentiation layer keeps the same network performance when we have 2 and 3 nodes in the network. For 6 nodes, we note a decrease of $15 \%$ when

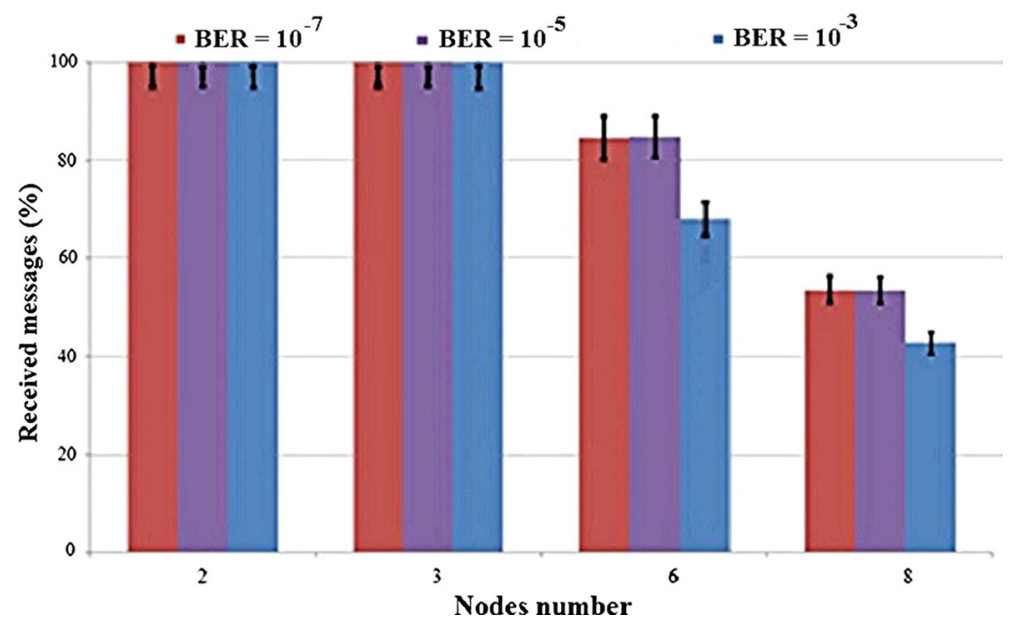

Fig. 14 Received messages percentage for different BER values 


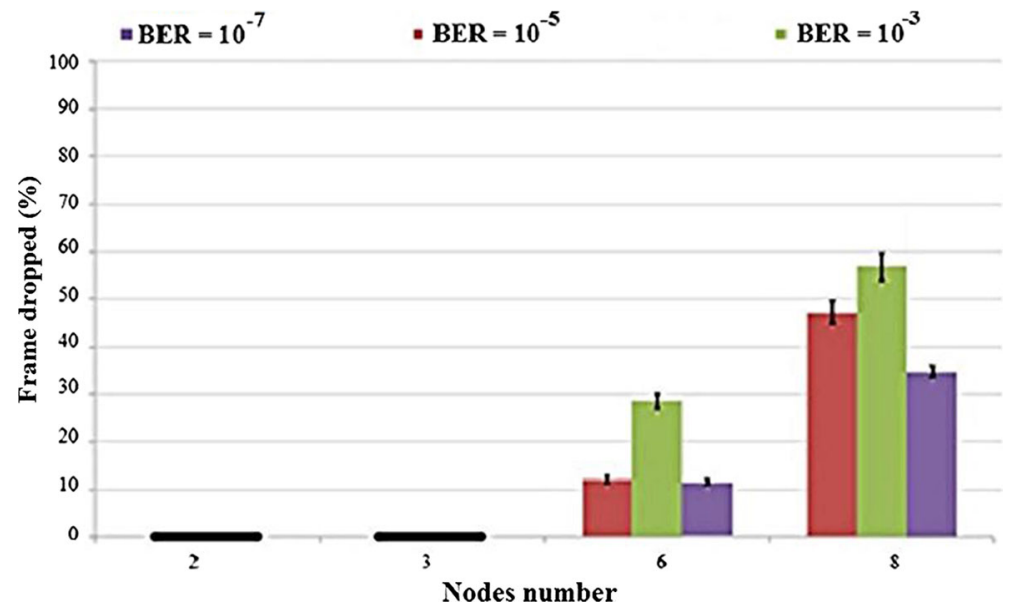

Fig. 15 Frame dropped percentage for different BER values

BER equal to $10^{7}$ and $10^{5}$ and $30 \%$ when BER equal to $10^{3}$. Our solution ensures at least the reception of $70 \%$ of messages.

For 8 nodes, the network performances degrade widely. The average percentage of received messages falls below $60 \%$ and under $40 \%$. Despite the difficult conditions, our solution may transmit at least $40 \%$ of messages.

Figure 15 shows that the queues overflow caused by the frames blockage due to retransmission problems or busy channel.

Figure 16 show that the value of BER has no major influence on our differentiation layer with purge function. The percentage of received messages (see Fig. 14) in the CFP decreased when BER equal to $10^{-3}$. The coordinator has received at least $80 \%$ of transmitted packets, which allows us to conclude that our differentiation layer with purge function resistant to the worst BER values.

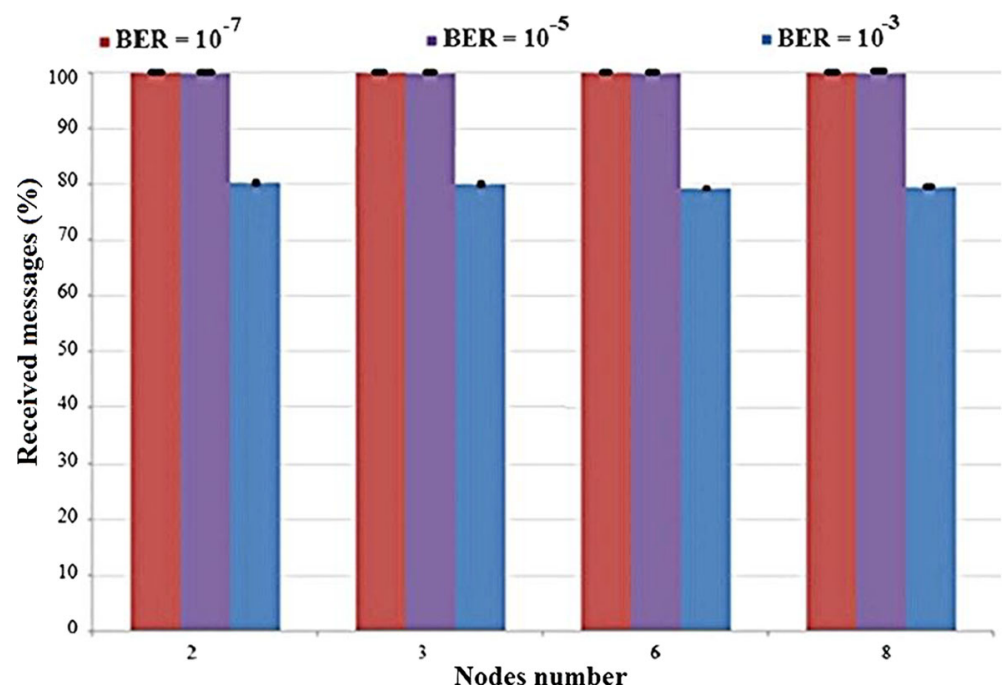

Fig. 16 Received messages percentage for different BER values 


\section{Conclusion}

In this paper, we introduced a new traffic differentiation architecture for WBAN based on IEEE 802.15.4. The first major issue is that, in our use case, the IEEE 802.15.4 do not explains how to use the CAP and CFP by the same node in the same super frame. We have chosen to use the purge function. The simulations performed as part of the study show that the use of the purge improves network performance. But, we have observed a slight performance degradation in the CAP. This function will be used by the differentiation layer in order to respect the different constraints defined by applications.

The simulation results show that differentiation layer allows a good management of transmission and ensuring the transmission of each frame in adequate period. The percentage of messages received in the CFP, reached $100 \%$, which shows the proper management of CAP and CFP.

To test the reliability of the differentiation layer, we introduced transmission error (realistic BER) in the simulator configuration. According to the results of simulation with BER equal to $10^{-7}$ and $10^{-5}$, we can conclude that our differentiation layer with purge is reliable under worst-case.

In the future work, we intend to compare our solution with real testbed based in WINo nodes $[38,39]$. We wish to add more canes and provide a data analysis algorithm of received information from the cane. It will be interesting to be more precise about the emergency case and add more specific algorithms and solutions with the consideration of the CPAM or cane breakdown. Also we think about the application of our solution and other solutions like video surveillance monitoring [40]. Finally we can test our proposition with other technologies like BLE, UWB, IEEE 802.15.6 or LoRaWAN.

\section{References}

1. Akyildiz, I. F., Su, W., Sankarasubramaniam, Y., \& Cayirci, E. (2002). Wireless sensor networks: A survey. Computer Networks, 38(4), 393-422.

2. Stankovic, J. (2008). When sensor and actuator networks cover the world. ETRI Journal, 30(5), 627-633.

3. Al Agha, K., Bertin, M. H., Dang, T., Guitton, A., Minet, P., Val, T., et al. (2009). Which wireless technology for industrial wireless sensors network? the development of ocari technology. IEEE Transactions on Industrial Electronics, 56(10), 13.

4. Noury, N., Herve, T., Rialle, V., Virone, G., Mercier, E., Morey, G., Moro, A. \& Porcheron, T. (2000). Monitoring behavior in home using a smart fall sensor and position sensors. In 1st annual international conference on microtechnologies in medicine and biology, 2000, pp. 607-610.

5. Schwiebert, L., Gupta, SKS., \& Weinmann, J. (2001). Research challenges in wireless networks of biomedical sensors. In Proceedings of the 7th annual international conference on mobile computing and networking, MobiCom '01, pp. 151-165.

6. Eysenbach, G. (2001). What is e-health? Journal of Medical Internet Research, 3(2), 20.

7. Bougeois, E., Van den Bossche, A., Cazenave, N., Redon, L., Soveja, A., Val, T. \& Villemur, T. (2012). Le projet CANet: une activit pluridisciplinaire liant recherche et pdagogie.

8. Val, T., Bougeois, E., Van den Bossche, A., Cazenave, N., Redon, L., Soveja, A. \& Villemur, T. (2013). Projet CANet: un systme de suivi de personnes mobilit rduite grce leur canne de marche, Magazine des IUT de France, $10 \& 11$.

9. http://www.ubitennis.com/english/2014/04/30/babolat-pure-drive-playintelligent-racquet-service/, babolat pure drive play: The intelligent racquet at your service. Accessed May 12, 2014.

10. http://indianexpress.com/article/sports/tennis/intelligent-racquets-to-be-thenew-tool-for-data-mining-intennis/, intelligent racquets to be the new tool for data mining in tennis. Accessed July 12, 2014

11. Pereira, V., Silva, JS. \& Monteiro, E. (2012). A framework for wireless sensor networks performance monitoring. In World of wireless, mobile and multimedia networks (WoWMoM), 2012. 
12. Lindh, T. \& Orhan, I. (2009). Wireless communication systems: Performance monitoring and control in contention-based wireless sensor networks. In ISWCS 2009.

13. Abreu, C., Miranda, F., Ricardo, M., \& Mendes, P. M. (2016). QoS-based management of biomedical wireless sensor networks for patient monitoring. SpringerPlus, 3, 239.

14. She, H., \& Lu, Z. (2007). Network-based system architecture for remote medical applications. AsiaPacic Advanced Network, China

15. Yoon, J. S., Gahng-Seop, A., Seong-Soon, J. \& al. (2010). PNP-MAC: Preemptive slot allocation and non-preemptive transmission for providing QoS in body area networks. In IEEE consumer communications and networking conference $(C C N C)$, pp. 1-15.

16. Hossien, M. (2010). A novel congestion control protocol for vital signs monitoring in wireless biomedical sensor networks. In IEEE WCNC.

17. Kwak, K. S. \& Ullah, S. (2010). A traffic-adaptive MAC protocol for WBAN. 2010 IEEE Globecom Workshops, Miami, FL, pp. 1286-1289.

18. George, S., Nikos, D., Rosario, S., Valeria, L., Giancarlo, F., \& Yiannis, A. (2016). Decentralized timesynchronized channel swapping for ad hoc wireless networks. IEEE Transactions on Vehicular Technology, 65(10), 8538-8553.

19. Galzarano, S., Fortino, G. \& Liotta, A. (2014). A learning-based mac for energy efficient wireless sensor networks. In Proceedings of the international conference on internet and distributed computing systems, pp. 396-406, Springer.

20. Hämäläinen, M., Iinatti, J., \& Mucchi, L. (2014). Wireless UWB body area networks: Using the IEEE802.15.4-2011. London: Academic Press.

21. Young Shin, S. (2013). A novel method for service differentiation in IEEE 802.15.4: Priority jamming. International Journal of Computers Communications Control, 8(1), 127-135.

22. Khssibi, S., Van den Bossche, A., Val, T., Idoudi, H., \& Azzouz Saidane, L. (2017). Optimization of IEEE 802.15.4: Overview theoretical study and simulation. International Journal of Information and Communication Technology, 10(2), 119-135.

23. Severino, R., Batsa, M., Alves, M. \& Koubaa, A. (2010). A traffic differentiation add-on to the IEEE 802.15.4 protocol: Implementation and experimental validation over a real-time operating system in DSD 2010. In Proceedings of the 2010, 13th Euromicro conference on digital system design: Architectures, methods and tools, France, pp. 501-508.

24. Saxena, N., Roy, A., \& Shin, J. (2008). Dynamic duty cycle and adaptive contention window based QoS-MAC protocol for wireless multimedia sensor networks. Computer Networks, 52(13), 2532-2542.

25. Firoze, A., Ju, L., \& Kwong, L. (2007). PR-MAC a priority reservation MAC protocol for wireless sensor networks. In ICEE 2007: Proceedings of the international conference on electrical engineering, Pakistan, pp. 1-6.

26. Xia, F., Li, J., Hao, R., Kong, X., \& Gao, R. (2013). Service differentiated and adaptive CSMA/CA over IEEE 802.15.4 for cyber-physical systems. The Scientific World Journal. https://doi.org/10.1155/2013/ 947808.

27. Kim, E., Kim, M., Youm, S. K., Choi, S., \& Kang, C. H. (2007). Priority-based service differentiation scheme for IEEE 802.15.4 sensor networks. AEU-International Journal of Electronics and Communications, 61(2), 69-81.

28. Youn, M. J., Oh, Y. Y., Lee, J. \& Kim, Y. (2007). IEEE 802.15.4 based QoS support slotted CSMA/CA MAC for wireless sensor networks. In SENSORCOMM 2007: International conference on sensor technologies and applications, Spain, pp. 113-117.

29. Koubaa, A., Alves, M., Nefzi, B., \& Song, Y. Q. (2006). Improving the IEEE 802.15.4 slotted CSMA, CA MAC for time-critical events in wireless sensor networks. In RTN, Proceedings of the workshop on real time networks, Germany, pp. 270-277.

30. Kim, T. H., \& Choi, S. (2006). Priority-based delay mitigation for event-monitoring IEEE 802.15.4 LRWPANs. IEEE Communications Letters, 10(3), 213-215.

31. Aykut Yigitel, M., Durmaz Incel, O., \& Ersoy, C. (2011). QoS-aware MAC protocols for wireless sensor networks: A survey. Computer Networks, 55(8), 1982-2004.

32. Nguyen, K., Nguyen, T., Chaing, C.K. \& Motani, M. (2006). A prioritized MAC protocol for multi-hop, event-driven wireless sensor networks. In ICCE 2006: Proceeding of the first international conference on communications and electronics, pp. 47-52.

33. Liu, Y., Elhanany, I. \& Qi, H. (2005). An energy-efficient QoS-aware media access control protocol for wireless sensor networks. In Proceedings of the IEEE international conference on mobile Adhoc and sensor systems conference, pp. 191.

34. Huang, Y. K., Pang, A. C., \& Hung, H. N. (2008). An adaptive GTS allocation scheme for IEEE 802.15.4. IEEE Transactions on Parallel and Distributed Systems, 9(5), 641-651. 
35. Villaverde, B. C., Rea, S. \& Pesch, D. (2010). D-SeDGAM: A dynamic service differentiation based GTS allocation mechanism for IEEE 802.15.4 WSN. In ITNG 2010: Proceedings of the 7th international conference on information technology: New generations, pp. 852-857.

36. Yuce, M. R. (2016). Ultra-wideband and $60 \mathrm{GHz}$ communications for biomedical applications. Berlin: Springer.

37. Khssibi, S. (2015). Using cane sensor networks for people monitoring applications, Ph.D., University of Jean Jaures.

38. Van den Bossche, A., Dalce, R. \& Val, T. (2016). OpenWiNo: An open hardware and software framework for fast-prototyping in the IoT. In International conference on telecommunications, Greece.

39. Lachtar, A., Val, T., \& Kachouri, A. (2016). 3DCane: A monitoring system for the elderly using a connected walking stick. International Journal of Computer Science and Information Security, 14(8), 535 .

40. Mohamed, A. B. H., Val, T., Andrieux, L., \& Kachouri, A. (2014). A help for assisting people based on a depth cameras system dedicated to elderly and dependent people. Journal of Biomedical Engineering and Medical Imaging, Society for Science and Education, 1(6), 51-56.

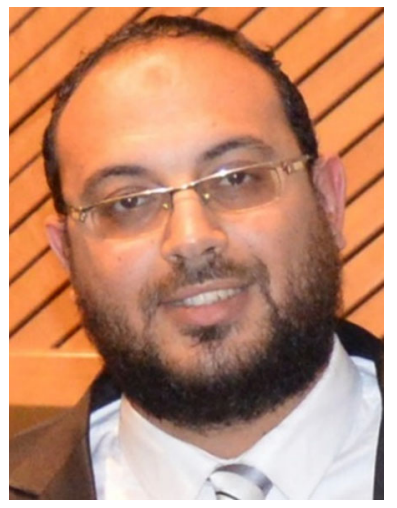

Sabri Khssibi is a Postdoctoral Researcher at University of Toulouse Jean Jaures since September 2016 and a member of IRIT-CNRS laboratory of Toulouse in IRT team. He earned his engineering and master degrees in computer science at the National School of Computer Science (ENSI), Tunisia in 2009 and 2010, respectively. He received his Ph.D degree jointly from University of Toulouse JEAN JAURES and ENSI, Tunisia (where he was also member of CRISTAL), in 2015. His current research focuses on issues related to wireless networking, sensor and mesh networks, quality of service (QoS), Device LayerInternet of Things (DL-IoT), cross-layer designs, etc.

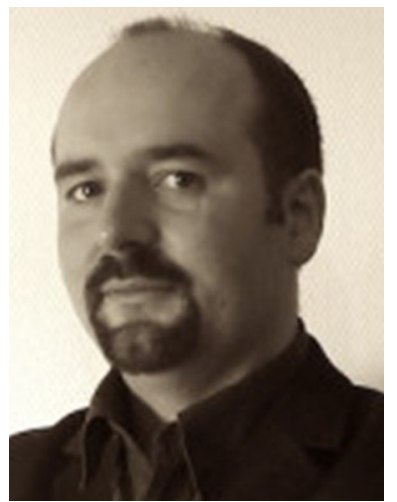

Adrien Van Den Bossche is an assistant professor at the University of Toulouse Jean Jaurès and a member of the IRT team of the Institut de Recherche en Informatique de Toulouse, France. He has obtained his $\mathrm{Ph} . \mathrm{D}$ in Computer Science in 2007. His research focuses on Medium Access Control (MAC) and localisation by Time-of-Flight (ToF) for wireless networks: ad-hoc/sensors/mesh networks and the IoT Device Layer (DL-IoT). He is involved in the Smart Home of Blagnac, a prototype house dedicated to maintaining the elderly at home. He teaches networking and embedded computing at the Institut Universitaire de Technologie de Blagnac, France. 


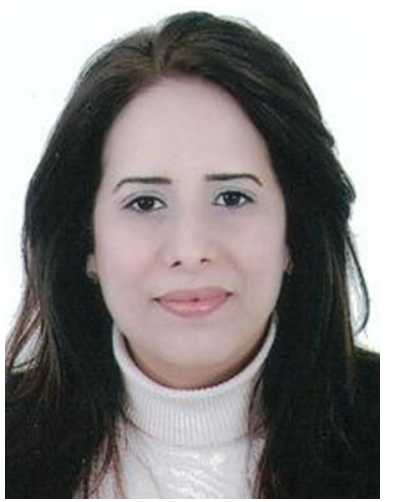

Hanen Idoudi is an associate professor in Computer Science at the National School of Computer Science, University of Manouba, Tunisia and a member researcher in the CRISTAL laboratory. She earned her engineering and master degrees in computer science both from the National School of Computer Science, Tunisia in 2001 and 2002, respectively. She received her Ph.D degree jointly from the same institution and University of Rennes 1, France (where she was also member of IRISA, INRIA, Rennes), in 2008. Her research focuses on issues related to wireless networking: ad hoc, sensor, mesh and cognitive radio networks, MAC optimization, networks modelling and performances evaluation, routing, quality of service (QoS), energy conservation and scheduling.

Leila Azouz Saidane is Professor at the National School of Computer Science (ENSI), at The University of Manouba, in Tunisia and the Chairperson of the PhD Commission at ENSI. She was the Director of this school and the supervisor of the Master's Degree program in Networks and Multimedia Systems. She is the co-director of RAMSIS group of CRISTAL Research Laboratory (Center of Research in Network and System Architecture, Multimedia and Image Processing) at ENSI. She collaborated on several international projects. She is author and co-author of several papers in refereed journals, magazines and international conferences.

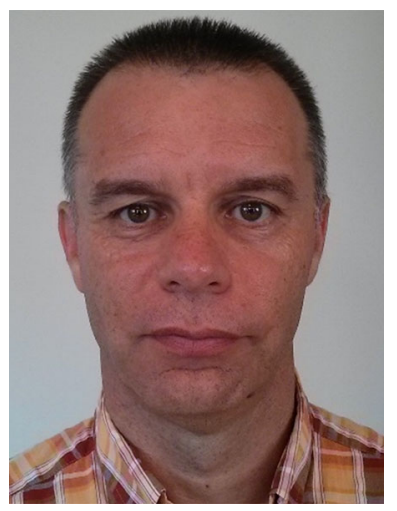

Thierry Val obtained his Ph.D. in computer science at Blaise Pascal University, Clermont-Ferrand, France, in 1993. In 1994, he became a lecturer at the University of Toulouse, where he currently teaches networks and computing systems. He obtained his HDR in 2002.He is now a professor for the University of Toulouse at the Blagnac Institute of Technology. He was sub-manager of the LATTIS laboratory, where he managed a research activity on wireless local networks and related protocols. He is now a member of IRIT-CNRS laboratory of Toulouse in IRT team. His current research focuses on wireless networks in smart homes, DL-IoT (Device Layer-Internet of Things). 